Research Article

\title{
Multiobjective Optimization of a Rotman Lens through the QLWS Minimization
}

\author{
Daniele Pinchera, ${ }^{1}$ Stefano Perna, ${ }^{2,3}$ and Marco Donald Migliore ${ }^{1}$ \\ ${ }^{1}$ DIEI-University of Cassino and Southern Lazio and ELEDIA@UNICAS, Via G. Di Biasio 43, 03043 Cassino, Italy \\ ${ }^{2}$ DI-University of Napoli "Parthenope", Centro Direzionale, Isola C4, 80143 Napoli, Italy \\ ${ }^{3}$ IREA-CNR, Via Diocleziano 328, 80124 Napoli, Italy
}

Correspondence should be addressed to Daniele Pinchera; pinchera@unicas.it

Received 10 August 2017; Revised 27 October 2017; Accepted 14 November 2017; Published 21 December 2017

Academic Editor: Shiwen Yang

Copyright (c) 2017 Daniele Pinchera et al. This is an open access article distributed under the Creative Commons Attribution License, which permits unrestricted use, distribution, and reproduction in any medium, provided the original work is properly cited.

\begin{abstract}
We address the multiobjective optimization of a Rotman lens by means of a recently proposed method based on the minimization of a properly defined global cost function named Quantized Lexicographic Weighted Sum (QLWS). More specifically, we have considered three different objectives concurring during the optimal synthesis of the lens. First, the difference between actual and desired delay among the excitations of the array elements fed by the lens needs to be lower than a given threshold. Second, gain losses of the beams scanned by the array fed by the lens need to be lower than a given threshold. Third, lens insertion losses should be as low as possible. Exploitation of the QLWS based approach allowed us to obtain in a few minutes a Rotman lens fulfilling these three concurring objectives and to improve the starting result obtained by a commercial software.
\end{abstract}

\section{Introduction}

The Rotman lens [1] is a planar structure implementing a true delay line beam-forming network (BFN) for linear arrays that are required to radiate different scanned beams within a given angular portion.

Due to practical relevance of Rotman lens, synthesis methods are a wide investigation subject. In particular, if one accepts that the delay among the excitations of the array elements fed by the lens is not perfectly linear for all the beams to be scanned, the lens synthesis gets easier. Indeed, in this case, it basically requires the optimal choice of just three specific geometric parameters of the focal arc where the input ports of the lens will be located [1]. In this regard, it is noted that the design of a Rotman lens involves the achievement of different concurring targets and/or constraints, such as the reduction of the lens insertion losses, the gain losses of the scanned beams, and the lens encumbrance. Accordingly, the optimal calculation of the three unknowns needed for the design of a Rotman lens generally requires solving a multiobjective problem [2-4]. However, although many papers have been written [5-13] with the aim of improving the lens architecture originally proposed by Rotman, just a few of them focus on the multiobjective nature of the lens synthesis.

In this work, we address such aspect. More specifically, we carry out a multiobjective optimization of a Rotman lens, by exploiting the method recently proposed in [14] for the synthesis of sparse arrays. In particular, the method is based on the minimization of a properly defined global cost function (GCF) inspired by the lexicographic order rules and named quantized lexicographic weighted sum (QLWS). In contrast to other optimization tools in electromagnetics [15-18], such QLWS-based approach allows to define a priori, in a simple and effective way, the priorities among the different optimization objectives, thus making it unnecessary to build cumbersome Pareto optimal fronts $[19,20]$.

In particular, we have considered three different optimization objectives. First, the difference between actual and desired delay among the excitations of the array elements fed by the lens needs to be lower than a given threshold. Second, gain losses of the beams scanned by the array fed by the 


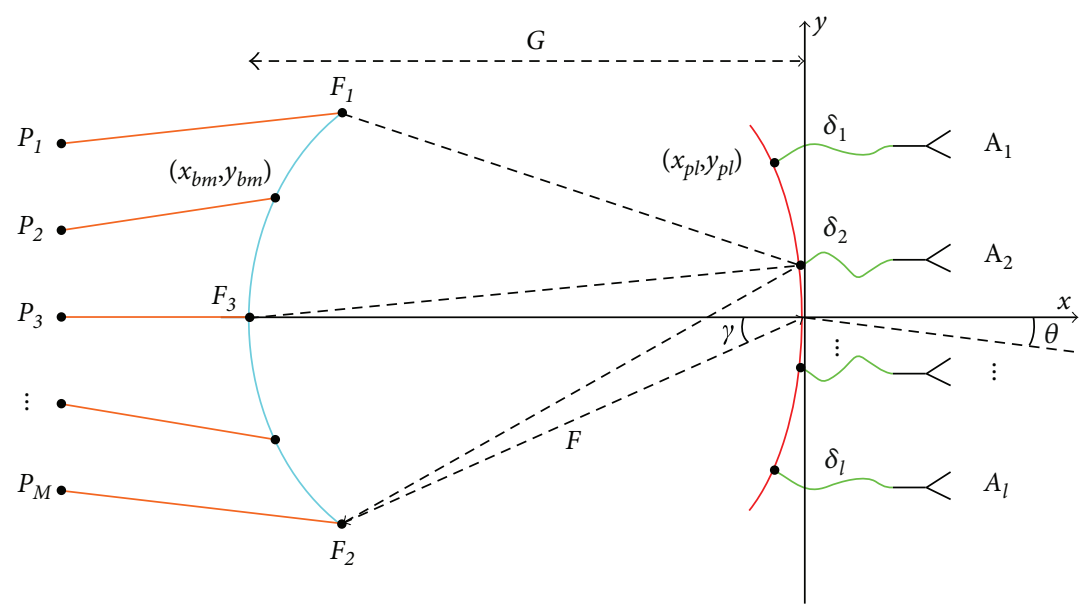

Figure 1: Geometry of the Rotman lens.

lens need to be lower than a given threshold. Third, lens insertion losses should be as low as possible. We started from the solution provided by a single-objective optimization tool implemented by a commercial software, and through the QLWS-based approach, in a few minutes, we improved it by obtaining a Rotman lens fulfilling all the three considered objectives.

\section{Synthesis of a Rotman Lens}

Let us briefly recall the basic principle of the Rotman lens design.

As a well-known design, this architecture allows the realization of a true delay line beam-forming network (BFN) for linear arrays by means of an extension of the Ruze lens [21] to a three focus architecture.

The basic scheme of the lens is depicted in Figure 1 for a linear array that is required to radiate $M$ different beams in the range $\theta \in[-\alpha, \alpha]$, being $\theta$ the angular deviation from the broadside direction. The considered array consists of $L$ equispaced antennas, say $A_{\ell}, \ell \in\{1, \ldots, L\}$. In the figure, the antennas are deployed along the $y$-axis: their positions are $\left(x_{0}, y_{\ell}\right), \ell \in\{1, \ldots, L\}$, with constant interelement distance $d$.

The lens consists of a set of $M$ input ports, denoted with $P_{m}, m \in\{1, \ldots, M\}$, and a set of $L$ output ports. The input ports are associated to the different $M$ scanned beams to be radiated, whereas the output ports are associated to the $L$ array antennas. The input ports are connected by transmission lines to corresponding $M$ ports, with coordinates $\left(x_{b m}, y_{b m}\right), m \in\{1, \ldots, M\}$, lying on the input curve depicted in cyan in the figure. The output ports, with coordinates $\left(x_{p \ell}, y_{p \ell}\right), \ell \in\{1, \ldots, L\}$, lie on the output curve, depicted in red in the figure, and are connected by transmission lines of electrical length $\delta_{\ell}, \ell \in\{1, \ldots, L\}$, to the corresponding array antennas.

In principle, the design of the lens would involve the calculation of $2 M+3 L$ unknowns represented by the port coordinates $\left(x_{b m}, y_{b m}\right), m \in\{1, \ldots, M\}$, on the input curve, the port coordinates $\left(x_{p \ell}, y_{p \ell}\right), \ell \in\{1, \ldots, L\}$, on the output curve, and the electrical lengths $\delta_{\ell}, \ell \in\{1, \ldots, L\}$. However, the number of the unknowns can be significantly reduced following the rationale in [1], where the input curve is built as a circular arc, called the focal arc, passing through three foci, say $F_{1}, F_{2}$, and $F_{3}$, with coordinates

$$
\begin{aligned}
& F_{1}=(-F \cos (\gamma), F \sin (\gamma)), \\
& F_{2}=(-F \cos (\gamma),-F \sin (\gamma)), \\
& F_{3}=(-G, 0),
\end{aligned}
$$

where the distances $F$ and $G$ and the angle $\gamma$ are highlighted in Figure 1. The three foci $F_{1}, F_{2}$, and $F_{3}$ in (1) identify three specific input ports: $F_{1}$ and $F_{2}$ are associated to the maximally scanned beams, that is, the beams to be radiated in the directions $\theta=\alpha$ and $\theta=-\alpha$, respectively, whereas $F_{3}$ is associated to the broadside beam $(\theta=0)$. Once the positions of $F_{1}, F_{2}$, and $F_{3}$ in (1) are set, the positions of the remaining $M-3$ input ports can be easily achieved by equispacing them on the focal arc between $F_{1}$ and $F_{2}$. The remaining $3 L$ unknowns represented by the length $\delta_{\ell}$ and the coordinates $\left(x_{p \ell}, y_{p \ell}\right)$ can be instead obtained by solving, for $\ell \in\{1, \ldots, L\}$, the following set of three equations:

$$
\begin{aligned}
& \operatorname{dist}\left(F_{1}, P_{p \ell}\right)+\delta_{\ell}+y_{\ell} \cos (\alpha)=F+\delta_{0}, \\
& \operatorname{dist}\left(F_{2}, P_{p \ell}\right)+\delta_{\ell}-y_{\ell} \cos (\alpha)=F+\delta_{0}, \\
& \operatorname{dist}\left(F_{3}, P_{p \ell}\right)+\delta_{\ell}=G+\delta_{0},
\end{aligned}
$$

where $\delta_{0}$ is an inessential offset term [1]. Note that these equations (2) enforce the condition that the foci $F_{1}$ and $F_{2}$ are associated to the maximally scanned beams and the focus $F_{3}$ to the broadside one.

Summing up, once the electrical array parameters (number $M$ of input ports, number $L$ of antennas, and maximum scanning angle $\alpha$ ) are defined, the overall lens design basically requires the proper choice for the three parameters $F, G$, and $\gamma$ in (1) and (2).

The drawback of this approach is that, at least in principle, only the input ports located in the three foci show a perfect linear delay among the excitations of all antenna 
elements. A deviation with respect to this ideal condition is instead expected for the other ports, which are associated to the beams other than the broadside and the maximally scanned ones. Accordingly, a quite obvious criterion for the choice of the three unknowns $F, G$, and $\gamma$ is to achieve a delay among the excitations of the antenna elements as linear as possible for all the input ports, in such a way to well radiate all the different $M$ scanned beams.

On the other side, it could be worth accounting for other concurring constraints and/or targets during the synthesis of a Rotman lens. First of all, to limit the gain losses when scanning the beam far away from the broadside direction, the field amplitude difference between the different scanned beams should be as low as possible. Furthermore, the lens insertion losses should be limited as much as possible. Finally, to meet the encumbrance and weight constraints that are typically enforced during the design of the overall antenna, the lens dimensions should be kept as small as possible. Of course, some other constraints could be required, as a specific range for the dimension of the ports, in order to ensure practical realizability. Accordingly, to obtain the optimal choice of the three parameters $F, G$, and $\gamma$ in (1) and (2), we have to deal with multiple concurring targets and different constraints. In other words, we have to solve a multiobjective ("multicriteria" or "multiparameter" or "multitarget") problem. To do this, in the following, we set a priori the priorities among the different concurring targets listed above and exploit the method recently proposed in [14] for the multiobjective synthesis of sparse arrays. The method, which is based on the minimization of a properly defined GCF named QLWS and inspired by the lexicographic order rules, is briefly recalled in the next section; for further details, the reader can refer to [14].

\section{Main Rationale of the QLWS Minimization Method}

Let us consider a generic multiobjective problem, where the $N$ optimization objectives, say $x_{k}, k \in\{1, \ldots, N\}$, are characterized by different $N$ levels of priorities, say $p_{k}, k$ $\in\{1, \ldots, N\}$, defined a priori. The approach in [14] addresses such kind of problem through the minimization of a GCF built as follows:

$$
\mathrm{QLWS}=\sum_{k=1}^{N} B^{p_{k}} \operatorname{ceil}\left((B-1) D_{k}\left(x_{k}\right)\right),
$$

where $B$ is a base number and ceil() takes the minimum integer greater or equal than its argument. Moreover, $D_{k}\left(x_{k}\right)$ is a function that maps each optimization objective $x_{k}$ onto a real number ranging within the interval $I=[0,1]$ : the smaller the value of $D_{k}\left(x_{k}\right)$, the closer the fulfilment of the target relevant to $x_{k}$. Some examples of $D_{k}\left(x_{k}\right)$ can be found in [14] and are not reported here for brevity.

The weighted sum in (3) is inspired by the lexicographic ordering rules, from which it retains its name, that is, quantized lexicographic weighted sum, briefly QLWS. Minimization of the QLWS in (3) has been introduced in [14] for the synthesis of sparse arrays; in particular, multiobjective global optimization was performed in that case. In the following section, we show that the QLWS can be exploited also to perform a multiobjective local optimization, as required for the synthesis of a Rotman lens.

\section{Numerical Results}

As an example, let us consider the multiobjective optimization of a Rotman lens for the beamforming of an array operating at a central frequency $f=9.6 \mathrm{GHz}$ and consisting of $L=16$ vertical $\lambda / 2$ wire dipoles, horizontally equispaced with interelement distance $d=\lambda / 2$. In particular, let us consider $M=16$ and $\alpha=\pi / 6$.

For such a kind of array, it can be shown that the scanning properties, even for the maximally scanned beam, are very marginally affected by the mutual coupling effects. Accordingly, such effects are not considered during the synthesis of the lens.

The lens is implemented in microstrip, with permittivity $\epsilon_{r}=2.2$ and substrate height $h_{\text {sub }}=0.5 \mathrm{~mm}$.

In our case, we consider the following three optimization objectives:

(i) Objective 1: the difference between actual and desired delay among the excitations of the array elements fed by the lens needs to be lower than a given threshold;

(ii) Objective 2: the gain losses of the beams scanned by the array fed by the lens need to be lower than a given threshold;

(iii) Objective 3: lens insertion losses should be as low as possible;

Moreover, as clarified in Section 3, to exploit the QLWS in (3), we have to set a priori the priorities among the different optimization objectives listed above. According to the considerations carried out in Section 2, we give the highest priority to objective 1 . This constraint can be coded by considering on one side the actual optical paths connecting the input ports and the antennas and on the other side the ideal paths that would ensure perfect linear delay among the excitations of the antenna elements. The maximum difference between these paths (maximum path error, briefly MPE) has to be as low as possible, say less that one-thousandth of wavelength. The subsequent level of priority is given to objective 2. In particular, we enforce that the amplitude variation between the beams (scan loss, briefly SL) must be less than $0.2 \mathrm{~dB}$. Finally, the lowest priority is given to objective 3 . In particular, we enforce that the insertion loss, briefly IL, should be as low as possible. These priorities are translated into the QLWS in (3) by exploiting the following settings:

(i) $N=3$ and $B=10^{4}$;

(ii) $p_{1}=2, D_{1}(M P E)$ built as in (4) with $\mathrm{MPE}_{\min }=10^{-3}$ $\lambda$ and $\mathrm{MPE}_{\max }=10^{-1} \lambda$;

(iii) $p_{2}=1, D_{2}(S L)$ built as in (4) with $\mathrm{SL}_{\min }=0.2 \mathrm{~dB}$ and $\mathrm{SL}_{\max }=10 \mathrm{~dB}$; 


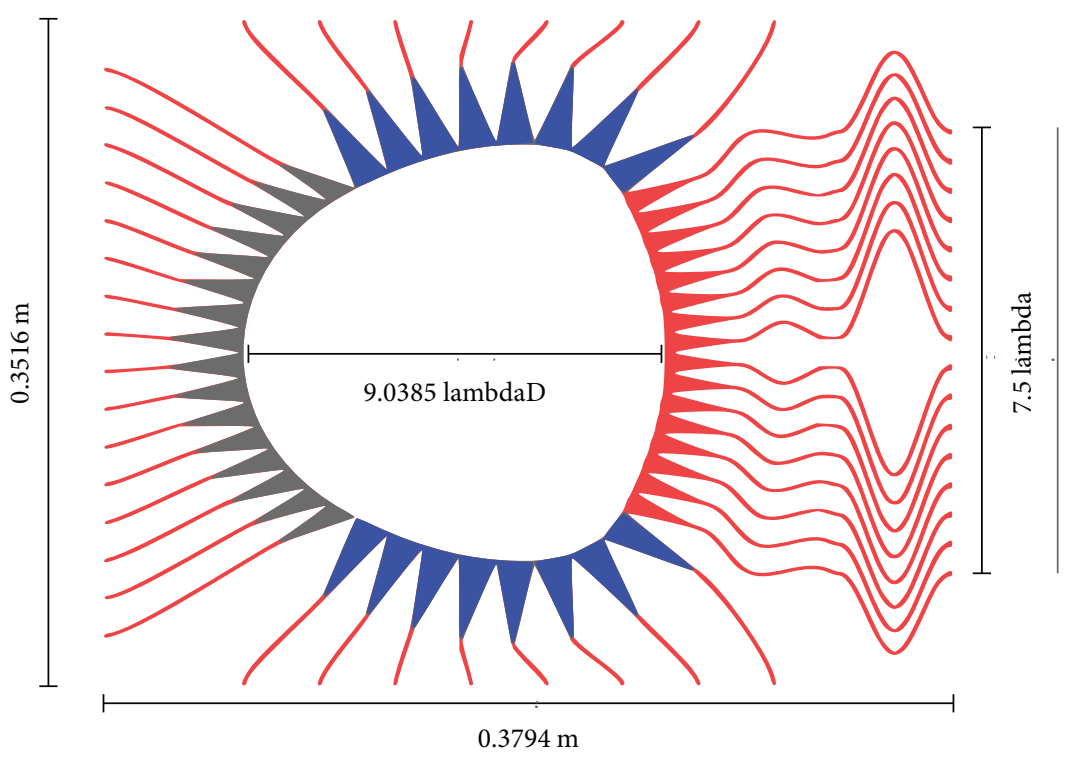

Figure 2: Lens obtained through RLD.

TABle 1: Parameters for the Rotman lens.

\begin{tabular}{lcc}
\hline & RLD & QLWS based optimization \\
\hline$F$ & $7.95 \lambda_{\mathrm{D}}$ & $8.31 \lambda_{\mathrm{D}}$ \\
$G$ & $9.04 \lambda_{\mathrm{D}}$ & $9.66 \lambda_{\mathrm{D}}$ \\
$\gamma$ & $30.00^{\circ}$ & $30.16^{\circ}$ \\
$\mathrm{MPE}$ & $2.50 \cdot 10^{-4} \lambda_{\mathrm{D}}$ & $1 \cdot 10^{-3} \lambda_{\mathrm{D}}$ \\
$\mathrm{SL}$ & $2.38 \mathrm{~dB}$ & $0.2 \mathrm{~dB}$ \\
$\mathrm{IL}$ & $7.179 \mathrm{~dB}$ & $4.193 \mathrm{~dB}$ \\
\hline
\end{tabular}

$\lambda_{\mathrm{D}}$ is the wavelength within the dielectric.

(iv) $p_{3}=0, D_{3}(I L)$ built as in (4) with $\mathrm{IL}_{\text {min }}=0 \mathrm{~dB}$ and $\mathrm{IL}_{\max }=10 \mathrm{~dB}$.

$$
D(x)=\left\{\begin{array}{cc}
1, \quad \text { if } x>x_{\max }, \\
0, \quad \text { if } x>x_{\min }, \\
\frac{x-x_{\min }}{x_{\max }-x_{\min }}, & \text { otherwise, }
\end{array}\right.
$$

As discussed in [14], the "ceiling" function in (3) is dropped for the lowest priority target. It is also remarked that the QLWS in (3) can be exploited by any available single-target optimization algorithm capable of handling discontinuities in the cost function. In particular, we exploited the Nelder-Mead optimization (implemented in the Matlab "fminsearch" function), which is a local search algorithm. Availability of a good starting point was thus needed to achieve better performances. In particular, as a starting solution, we have considered a layout automatically obtained by means of the Rotman lens designer (RLD) software by Remcom. Such a layout is shown in Figure 2, and its main parameters are reported in Table 1 . The benchmarking of the lenses has been obtained by means of a custom-built ray tracing code, developed following the guidelines in [22]. From Table 1, we understand that the starting lens satisfies the requirement on the MPE, but it fails to satisfy the requirement on the SL. Differently, exploitation of the proposed QLWS in (3) within the Nelder-Mead algorithm, along with the use of the same custom-built ray tracing code, leads to an optimized solution (shown in Figure 3) that fully satisfies both the requirements on the MPE and on the SL. Also, it guarantees a much lower IL $(4.193 \mathrm{~dB}$ with respect to $7.179 \mathrm{~dB})$.

To achieve a deeper insight on the used QLWS, we show in Figure 4 its evolution during the implemented optimization. In particular, the blue curve represents the value of the QLWS for each calling during the Nelder-Mead minimization, whereas the red curve represents the minimum achieved cost. Finally, in the figure, we have superimposed three bands. The yellow one marks the region QLWS $>=B^{2}$, where all the three considered optimization objectives are not fulfilled; see the expression of (3) with our setting. The pink band marks the region $B<=$ QLWS $<B^{2}$, where only objective 1 is fulfilled; see again (3). Finally, the green band marks the region $0<$ QLWS $<B$, where both objective 1 and objective 2 are reached, and the algorithm tries to pursue at best objective 3; see again (3). In Table 2, we have reported some noticeable points from the red curve plotted in Figure 4. It can be seen that at iteration 1, that is, the starting solution, only objective 1 is reached, since the QLWS belongs to the pink band. At iteration 66, the QLWS still belongs to the pink band, but it approaches the green one: this means again that only objective 1 is satisfied; however, objective 2 is very close to being reached. As can be seen, this happens at iteration 67, where the QLWS jumps onto the green band. In the following iterations, as expected, the algorithm acts in order to reduce as much as possible the IL. Note that at iteration 200 an improvement of the IL is achieved with respect to iteration 67.

It is remarked that due to the exploitation of the QLWS in (3), the different objectives considered in our synthesis have been reached at different iterations according to the priorities 


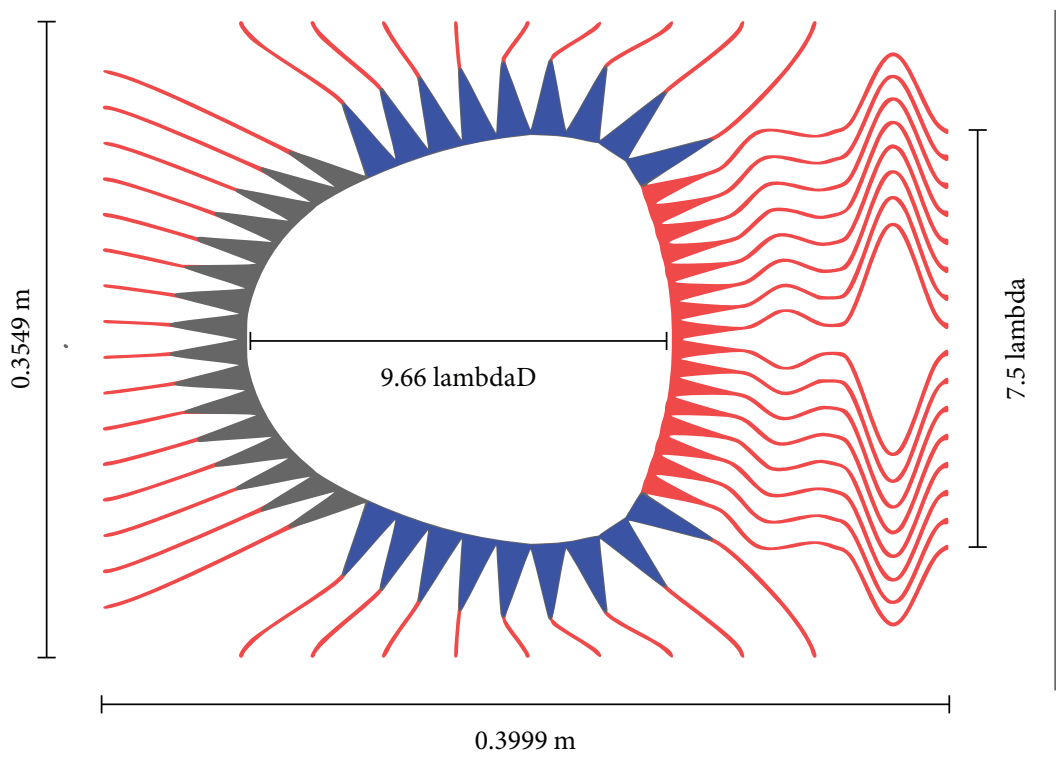

FIGURE 3: Lens obtained through the QLWS-based optimization.

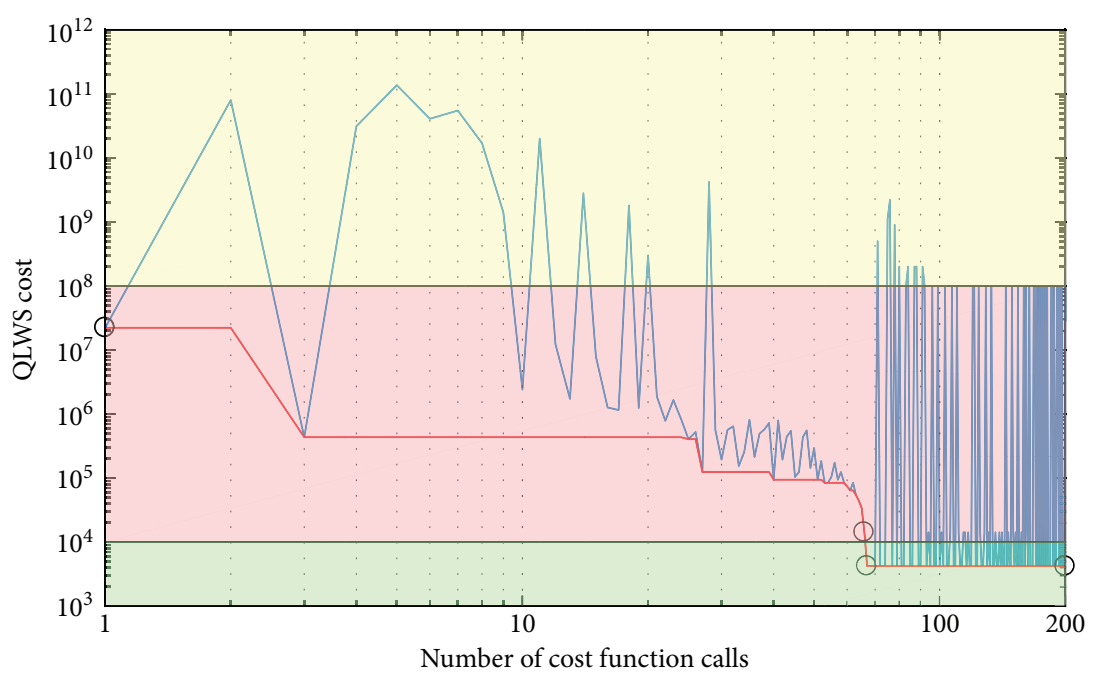

FIgURE 4: Behaviour of the QLWS cost function for the Rotman lens optimization. Blue line: QLWS cost calculated for each call in the Nelder Mead algorithm. Red line: minimum cost found by the algorithm. The points marked with black circles are detailed in Table 2.

TABLE 2: Behaviour of the Rotman lens parameters for some of the noticeable points of Figure 4.

\begin{tabular}{lcccc}
\hline iter. number & MPE $(\lambda / 1000)$ & SL $(\mathrm{dB})$ & IL $(\mathrm{dB})$ & QLWS \\
\hline 1 & 0.25027 & 2.3779 & 7.1791 & $2223^{\prime} 7178.370$ \\
66 & 0.79712 & 0.20076 & 4.2229 & $1^{\prime} 4222.439$ \\
67 & 0.76482 & 0.19931 & 4.2248 & 4224.334 \\
200 & 1 & 0.2 & 4.1929 & 4192.463 \\
\hline
\end{tabular}

that we have set a priori. No one told the algorithm to make a "switch" on what it was searching; such an information is already coded in the QLWS.

As it is evident from Figures 2 and 3, the electrical schemes of the two lenses obtained before and after the exploitation of the proposed QLWS are very similar. Notwithstanding, little local variations of the lens parameters allow the fulfilment of the constraints, which was not possible with the starting layout automatically provided by the used commercial software. It is remarked that the overall optimization took less than five minutes on a i5-2310 personal computer.

The last consideration is finally needed on the accuracy of the presented results. The three considered parameters have been calculated during the optimization by means of fast high-frequency approximation algorithms. If a better accuracy is needed in the calculation of these parameters, the QLWS approach could be employed as well, by calling within the optimizer EM solvers based on full-wave methods. This, of course, would involve a longer optimization time. 


\section{Conclusions}

In this work, we have discussed the multiobjective optimization of a Rotman lens, by means of a relatively novel cost function, the quantized lexicographic weighted sum (QLWS). This function allows the solution of multicriteria problems, handling the optimization constraints according to their priorities.

Such QLWS has recently been proposed to address the multiobjective global optimization related to the synthesis of sparse arrays. We have now demonstrated that by its usage it is possible to perform also multiobjective local optimization.

In particular, the use of the QLWS, together with the Nelder-Mead optimizer, allowed to significantly improve the design solution of a Rotman lens provided by a commercial software. Also, the obtained final layout correctly satisfied all the design specifications.

It is worth noting that the presented approach is one of the few that allows a local multicriteria optimization, and the presented results confirm the effectiveness of the lexicographic approach that allows the translation of the specifications of the problem in a simple and direct way.

\section{Conflicts of Interest}

The authors declare that there is no conflict of interests regarding the publication of this paper.

\section{Acknowledgments}

This work was partially developed within a research contract, signed in 2015, between the University of Napoli "Parthenope" and the University of Cassino and Southern Lazio.

\section{References}

[1] W. Rotman and R. Turner, "Wide-angle microwave lens for line source applications," IEEE Transactions on Antennas and Propagation, vol. 11, no. 6, pp. 623-632, 1963.

[2] R. T. Marler and J. S. Arora, "Survey of multi-objective optimization methods for engineering," Structural and Multidisciplinary Optimization, vol. 26, no. 6, pp. 369-395, 2004.

[3] R. C. Hansen, "Design trades for Rotman lenses," IEEE Transactions on Antennas and Propagation, vol. 39, no. 4, pp. 464-472, 1991.

[4] S. Vashist, M. K. Soni, and P. K. Singhal, "A review on the development of Rotman lens antenna," Chinese Journal of Engineering, vol. 2014, Article ID 385385, 9 pages, 2014.

[5] T. Katagi, S. Mano, and S. Sato, "An improved design method of Rotman lens antennas," IEEE Transactions on Antennas and Propagation, vol. 32, no. 5, pp. 524-527, 1984.

[6] D. R. Gagnon, "Procedure for correct refocusing of the Rotman lens according to Snell's law," IEEE Transactions on Antennas and Propagation, vol. 37, no. 3, pp. 390-392, 1989.

[7] P. K. Singhal, P. C. Sharma, and R. D. Gupta, "Rotman lens with equal height of array and feed contours," IEEE Transactions on Antennas and Propagation, vol. 51, no. 8, pp. 20482056, 2003.

[8] R. Uyguroglu and A. Y. Oztoprak, "A method for minimizing the phase errors of Rotman lenses," in 2009 International
Conference on Electrical and Electronics Engineering - ELECO 2009, pp. II-174-II-176, Bursa, Turkey, 2009.

[9] R. P. S. Kushwah and P. K. Singhal, "Design of 2d-bootlace lens with five focal feed for multiple beam forming," Journal of Electromagnetic Analysis and Applications, vol. 3, no. 2, pp. 39-42, 2011.

[10] W. Zongxin, X. Bo, and Y. Fei, "A multibeam antenna array based on printed Rotman lens," International Journal of Antennas and Propagation, vol. 2013, Article ID 179327, 6 pages, 2013

[11] A. Attaran and S. Chowdhury, "Fabrication of a $77 \mathrm{GHz}$ Rotman lens on a high resistivity silicon wafer using lift-off process," International Journal of Antennas and Propagation, vol. 2014, Article ID 471935, 9 pages, 2014.

[12] Y. Liu, H. Yang, S. Mao, and J. Zhu, "A multibeam dual-band orthogonal linearly polarized antenna array for satellite communication on the move," International Journal of Antennas and Propagation, vol. 2015, Article ID 102959, 8 pages, 2015.

[13] M. Rajabalian and B. Zakeri, "Optimisation and implementation for a non-focal Rotman lens design," IET Microwaves, Antennas \& Propagation, vol. 9, no. 9, pp. 982-987, 2015.

[14] D. Pinchera, S. Perna, and M. D. Migliore, "A lexicographic approach for multi-objective optimization in antenna array design," Progress In Electromagnetics Research $M$, vol. 59, pp. 85-102, 2017.

[15] Y. Rahmat-Samii and E. Michielssen, "Electromagnetic optimization by genetic algorithms," Microwave Journal, vol. 42, no. 11, p. 232, 1999.

[16] A. Hoorfar, "Evolutionary programming in electromagnetic optimization: a review," IEEE Transactions on Antennas and Propagation, vol. 55, no. 3, pp. 523-537, 2007.

[17] M. Donelli, D. Franceschini, P. Rocca, and A. Massa, “Threedimensional microwave imaging problems solved through an efficient multiscaling particle swarm optimization," IEEE Transactions on Geoscience and Remote Sensing, vol. 47, no. 5, pp. 1467-1481, 2009.

[18] P. Rocca, G. Oliveri, and A. Massa, "Differential evolution as applied to electromagnetics," IEEE Antennas and Propagation Magazine, vol. 53, no. 1, pp. 38-49, 2011.

[19] T. Goel, R. Vaidyanathan, R. T. Haftka, W. Shyy, N. V. Queipo, and K. Tucker, "Response surface approximation of Pareto optimal front in multi-objective optimization," Computer Methods in Applied Mechanics and Engineering, vol. 196, no. 4-6, pp. 879-893, 2007.

[20] K. Deb, "Multi-objective optimization," in Search Methodologies, pp. 403-449, Springer, Boston, MA, USA, 2014.

[21] J. Ruze, "Wide-angle metal-plate optics," Proceedings of the IRE, vol. 38, no. 1, pp. 53-59, 1950.

[22] J. Dong and A. I. Zaghloul, "Hybrid ray tracing method for microwave lens simulation," IEEE Transactions on Antennas and Propagation, vol. 59, no. 10, pp. 3786-3796, 2011. 


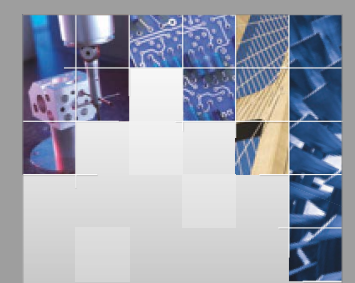

\section{Enfincering}
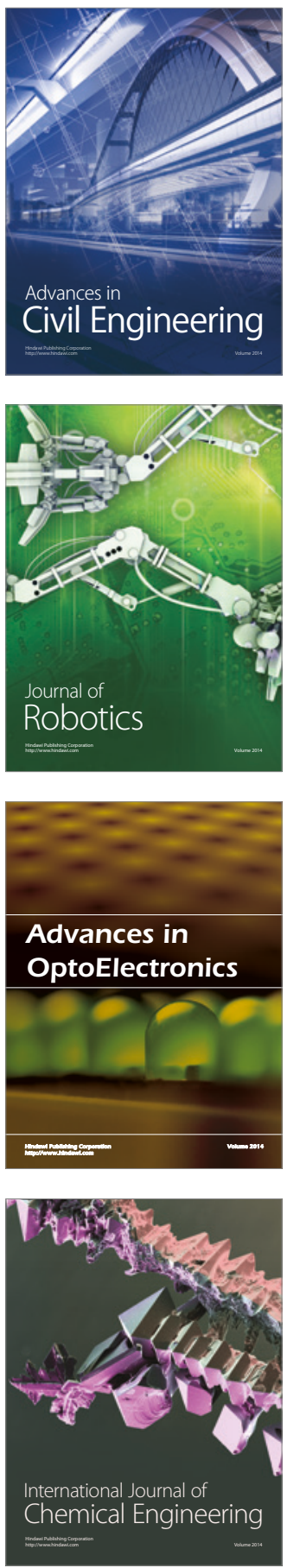

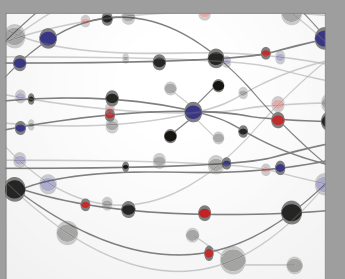

The Scientific World Journal

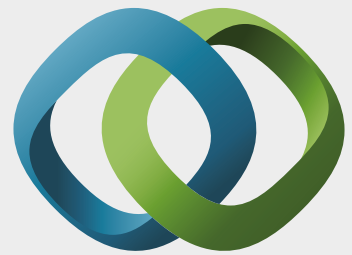

\section{Hindawi}

Submit your manuscripts at

https://www.hindawi.com
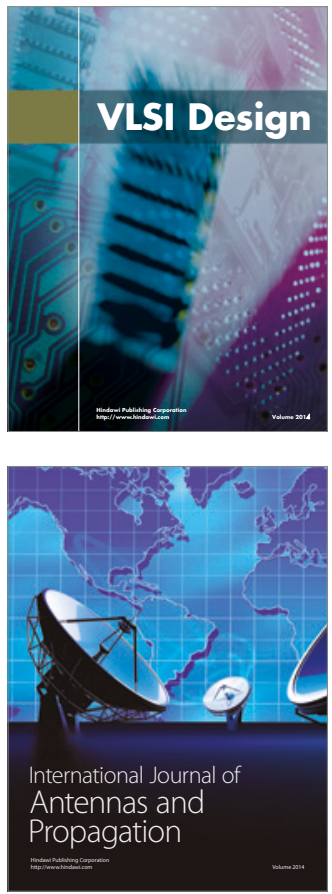

\section{Rotating}

Machinery
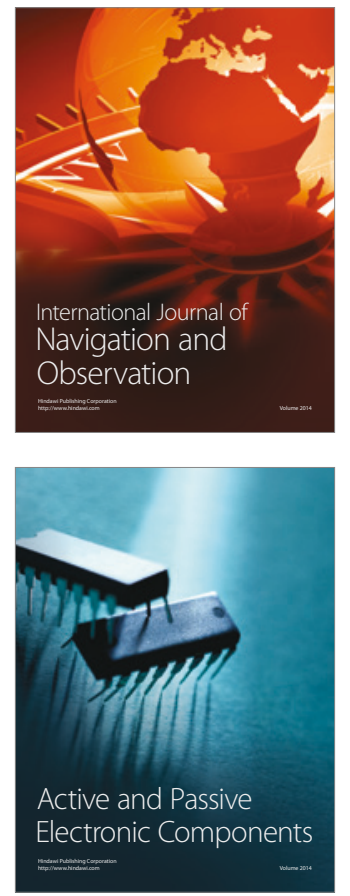
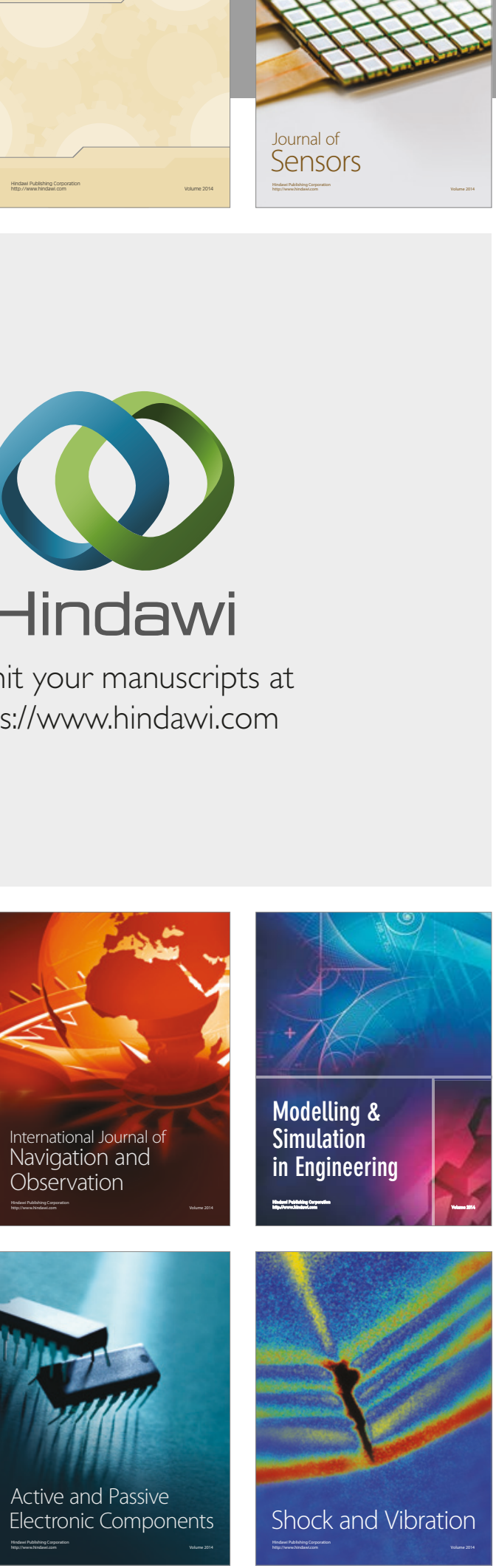
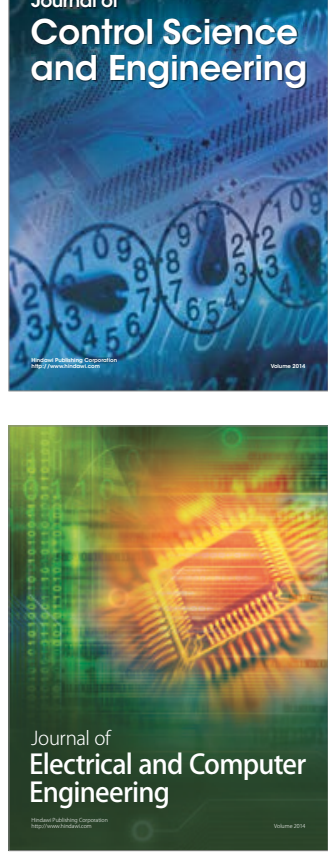

Distributed

Journal of

Control Science

and Engineering
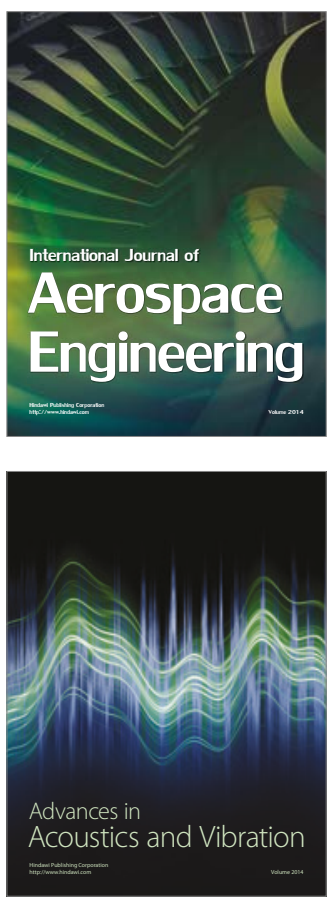

Sensor Networks 